\title{
Retinitis Pigmentosa Sine Pigmento Masquerading as Normal Tension Glaucoma
}

\author{
Justin MK Tong, Carmen KM Chan, Dexter YL Leung \\ Department of Ophthalmology and Visual Sciences, The Chinese University of Hong Kong, Hong Kong Eye Hospital \\ Hong Kong
}

\section{INTRODUCTION}

Retinitis pigmentosa (RP) is a heterogeneous group of diseases with progressive photoreceptor degeneration. In some RP patients, the typical bone spicule formation is lacking and the retina may appear normal in the early stages of the disease. ${ }^{1}$ This poses a diagnostic challenge to the ophthalmologist. We herein report a case of unilateral progressive visual field loss and optic atrophy due to RP sine pigmento which was initially masqueraded as normal tension glaucoma (NTG).

\section{CASE REPORT}

A 42 years-old gentleman was referred to our unit with progressive blurring of vision in his right eye for 6 months, especially affecting peripheral vision and in dim conditions. He had a history of hypertension, was an exsmoker, with no family history of glaucoma/Retinitis pigmentosa (RP). Initial examination revealed a visual acuity of 6/6 and normal color vision OU, with no relative afferent pupillary defect. Autorefraction yielded - 6.50 DS (OD) and -7.50 DS (OS). The intraocular pressure (IOP) by applanation tonometry was $17 \mathrm{~mm} \mathrm{Hg}(\mathrm{OD})$ and $18 \mathrm{~mm}$ $\mathrm{Hg}(\mathrm{OS})$. Fundal exam showed myopic fundi OU with pink disks and vertical cup-to-disk ratios of 0.6 (OD) and 0.5 (OS). There was inferior rim thinning and peripapillary atrophy of the right optic disk (Figs 1A and B). Examination was otherwise unremarkable. Central corneal thickness was $554 \mu \mathrm{m}$ (OD) and $567 \mu \mathrm{m}$ (OS). Automated white-on-white threshold perimetry (program 24- 2, SITA fast, model 750, Humphrey Instruments, Dublin, CA) showed right constricted field (superior > inferior) extending in an arcuate fashion from the blind spot (Fig. 2A). Optical coherence tomography showed a borderline- thinned peripapillary retinal nerve fiber layer thickness of $88.75 \mu \mathrm{m}$ OD and $90.89 \mu \mathrm{m}$ OS. Magnetic resonance imaging of the brain, orbit and pituitary gland with contrast was unremarkable. In view of right inferior optic disk rim thinning and normal IOP together with corresponding superior constricted visual field, normal tension glaucoma (NTG) was suspected. However, as the severity of the visual field loss was out of proportion to the structural damage (mean deviation $=-15.66 \mathrm{~dB}$ ) and a repeat visual field examination one year after presentation revealed a rapid mean deviation decline of $-7.99 \mathrm{~dB}$ (Fig. 2B), a review by medical retinal specialist was initiated. At this point, his visual acuity and color vision remained normal OU but there was a right-sided relative afferent pupillary defect, with progressive changes in the retina (Fig. 1B). The left eye remained normal. Full field electroretinogram (ERG) of his right eye showed nonrecordable scotopic rod responses. For the photopic cone
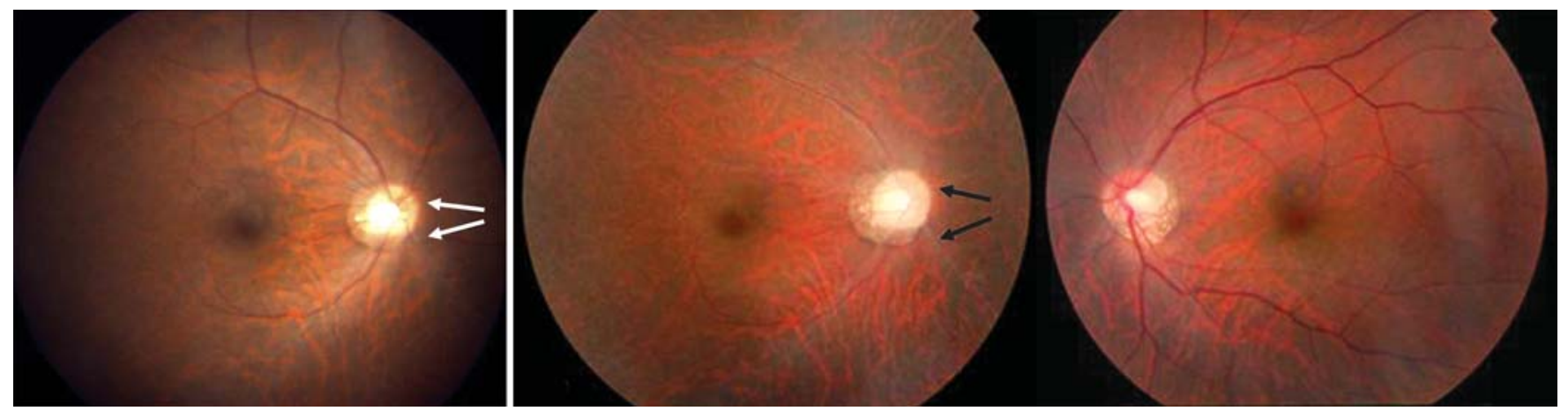

Figs 1A and B: (A) Fundus photograph from April 2006 showed enlarged vertical cup-to-disk ratio without attenuation of retinal vessels (white arrows) nor intraretinal pigmentation. (B) Fundus photograph from September 2007 showed disk pallor, narrowing of retinal vessels (black arrows) with diffuse subtle retinal pigmentary changes. The left eye appeared normal except for peripapillary atrophy (bottom left) 


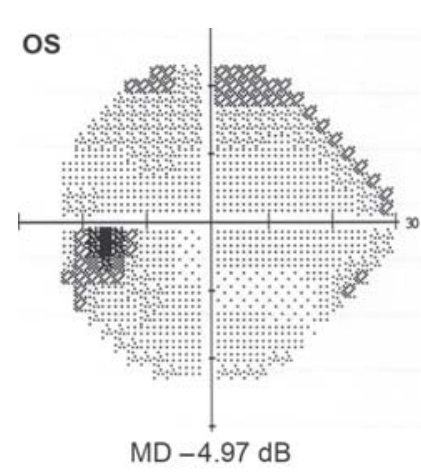

Fig. 2A: Visual field (Humphrey SITA fast 24-II) at presentation showing superior $>$ inferior arcuate defects in the right eye

responses, the a-wave was nonrecordable and the b-wave was delayed with a smaller amplitude than normal. The $30 \mathrm{~Hz}$ response also had a reduced amplitude. The left eye responses were normal. The final diagnosis was thereby confirmed by the ERG finding.

\section{DISCUSSION}

We initially considered NTG as our provisional diagnosis owing to an enlarged vertical cup-to-disk ratio, inferior disk rim thinning and corresponding visual field loss, although the highly asymmetrical involvement was not typical and the structuralfunctional relation was out of proportion. With time, there was a rapid decline of visual field without significant changes in media opacity (mean deviation deteriorated $-7.99 \mathrm{~dB}$ in a year). These made the diagnosis of NTG less likely. Another diagnostic clue in this case is the progressive constriction of visual field despite adequate IOP reduction. RP is usually a bilateral disease, but unilateral cases have been reported., ${ }^{1,2}$

We have also documented, from our patient, that progression from "normal" fundal appearance to a phase with vessel attenuation, disk pallor and rapid visual field progression can occur within a year's time for unilateral RP sine pigmento.

In summary, RP sine pigmento should be considered as a differential diagnosis in young NTG suspects who had out-ofproportion structure-function correlation, even without a family
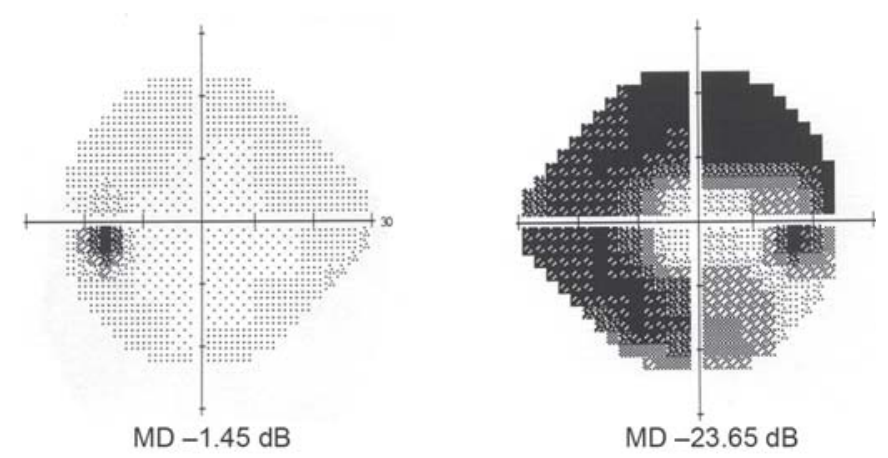

Fig. 2B: Visual field one year later, showing deterioration in the right eye

history or obvious retinal pigment epithelium abnormalities such as bone spicule formation. An ERG is helpful to confirm the diagnosis in such cases.

\section{REFERENCES}

1. Jacobson JH and Stephens G. Unilateral retinitis pigmentosa sine pigmento. Arch Ophthalmol 1962; 67:456-58.

2. Spadea L, Magni R, Rinaldi G, Dragani T and Bianco G. Unilateral retinitis pigmentosa: Clinical and electrophysiological report of four cases. Ophthalmologica 1998; 212:350-54.

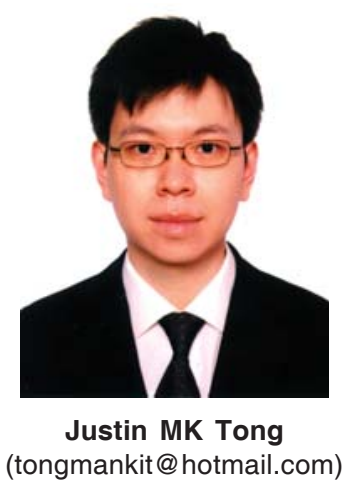

